\title{
AVALIAÇÃO DO MONITORAMENTO METACOGNITIVO A PARTIR DO JULGAMENTO DE DESEMPENHO DE ADULTOS EM PROCESSO DE ALFABETIZAÇÃO
}

\author{
Márcia Akemi Fujie \\ Universidade Federal de São Carlos \\ Patrícia Waltz Schelini \\ Universidade Federal de São Carlos
}

\begin{abstract}
Resumo
A metacognição, a capacidade do indivíduo em autorregular seus processos cognitivos, engloba o monitoramento metacognitivo, pelo qual o sujeito acompanha e avalia seu desempenho cognitivo. 0 presente estudo teve como finalidade investigar o monitoramento metacognitivo de adultos em processo de alfabetização por meio do julgamento de desempenho em tarefas cognitivas, assim como observar possíveis correlações entre desempenho real e estimado dos participantes. Participaram do estudo 15 adultos de um programa de alfabetização, de ambos os gêneros e com idades entre 20 e 74 anos. Os materiais aplicados foram: Entrevista Inicial; Teste R-1 e subtestes Código, Procurar Símbolos e Dígitos do WAIS III; Registro de Julgamentos de 0 a 10 e Registro de Julgamento a partir de um objeto de medida. Resultados indicaram correlação significativa entre os escores brutos de desempenho real dos subtestes Códigos, Procurar Símbolos e Dígitos com seus julgamentos de desempenho a partir de um objeto de medida.
\end{abstract}

Palavras-chave: alfabetização; medidas; metacognição; testes psicológicos.

\section{METACOGNITIVE MONITORING ASSESSMENT THROUGH JUDGEMENT EVALUATION OF ADULTS IN LITERACY DEVELOPMENT}

\begin{abstract}
Metacognition, the individual's capability to monitor and self-regulate their own cognitive process, encompasses the metacognitive monitoring, a process in which the individual follows their own cognitive performance. The present study aimed to investigate the metacognitive monitoring in adults in process of alphabetization, according to their judgement of performance on cognitive tasks, and to observe possible correlations between real and estimated performance by the participants. The study consisted of 15 adults in a literacy program, of both genders and with ages ranging from 20 to 74 . The materials applied were: Initial Interview; R-1 Test and subtests Digit Symbol-Coding, Symbol Search and Digit Span from WAIS III; Judgement Register from 0 to 10 and Judgement Register using a measurement object. Results indicated a significant correlation between the real performance of the subtests Digit Symbol-Coding, Symbol Search and Digit Span with their estimated performance from the judgement with a measurement object.
\end{abstract}

Keywords: literacy; measurement; metacognition; psychological testing. 


\title{
EVALUACIÓN DEL MONITOREO METACOGNITIVO A PARTIR DEL JUICIO DE DESEMPEÑO DE ADULTOS EN PROCESO DE ALFABETIZACIÓN
}

\begin{abstract}
Resumen
La metacognición, la capacidad del individuo en autorregular sus procesos cognitivos, abarca el monitoramiento metacognitivo, en el cual el sujeto acompaña y evalúa su desempeño cognitivo. El presente estudio tuvo como objetivo investigar el monitoramiento metacognitivo en adultos en proceso de alfabetización por medio del juicio de desempeño en tareas cognitivas, así como observar posibles correlaciones entre desempeño real y estimado de los participantes. En el estudio participaron 15 adultos de un programa de alfabetización, de ambos géneros y con edades entre 20 y 74 años. Los materiales aplicados fueron: Entrevista Inicial; Prueba R-1 y subtestes Código, Buscar Símbolos y Dígitos de WAIS III; Registro de Juicios de 0 a 10 y Registro de Juicio a partir de un objeto de medida. Los resultados indicaron una correlación significativa entre los puntajes brutos de rendimiento real de las subpruebas Códigos, Buscar Símbolos y Dígitos con sus juicios de rendimiento a partir de un objeto de medida.
\end{abstract}

Palabras clave: alfabetización; medidas; metacognición; tests psicológicos.

\section{INTRODUÇÃO}

A metacognição, entendida como uma das subdivisões no estudo da cognição, foi introduzida por Flavell, no início da década de 1970, como a habilidade de se pensar sobre o pensar, ou, mais especificamente, a habilidade do indivíduo em monitorar e autorregular seus processos cognitivos, de forma a refletir sobre suas próprias considerações e cognições (Flavell, 1987; França \& Schelini, 2018; Jou \& Sperb, 2006; Reynolds \& Wade, 1986). Essa concepção caracterizou o indivíduo como um aprendiz ativo, racional e reflexivo na compreensão adequada de como a aprendizagem, a memória e o pensamento funcionam, ressaltando sua habilidade e consciência sobre o monitoramento e o controle de suas atividades cognitivas enquanto empenhados na leitura, compreensão de textos, estudo, memória e resolução de problemas (Brown, 1978; Flavell \& Wellman, 1977; Reynolds \& Wade, 1986; Weinert \& Kluwe, 1987).

As propostas de Flavell ampliaram o conceito de metacognição, partindo do simples conhecimento sobre a própria cognição para a sugestão de que tal conhecimento pode ser empregado pelo indivíduo para planejar, monitorar, regular e avaliar suas próprias atividades cognitivas, como a aprendizagem, compreensão, raciocínio e resolução de problemas (Jou \& Sperb, 2006; Woolfolk, 2000). O planejamento das atividades cognitivas estabelece a melhor forma para realizar determinada tarefa, como, por exemplo, a quantidade de tempo despendida para sua realização, por onde começar ou quais as partes que mais exigem empenho. $O$ monitoramento acompanha o desempenho enquanto a atividade é realizada, informando, avaliando e relacionando o desempenho obtido com as estratégias escolhidas e utilizadas, de forma a verificar o grau de adequação das estratégias exploradas durante a tarefa, possibilitando a autonomia e consciência do indivíduo na elaboração de seu próprio conhecimento, assim como a inferência de ações fundamentais para a resolução 
de problemas, e possivelmente modificações em seus processos cognitivos (Dal'evedove, Neves, \& Fujita, 2014; Lima Filho \& Bruni, 2015; Tanikawa \& Boruchovitch, 2016; Woolfolk, 2000).

Diferentes técnicas podem ser utilizadas para a avaliação do monitoramento metacognitivo. Desoete (2008) nomeia duas delas: avaliação online e off-line. As avaliações on-line ocorrem durante a realização das tarefas, enquanto o sujeito exercita tanto suas habilidades cognitivas quanto metacognitivas (Desoete, 2008; Schelini et al., 2016). Schelini et al. (2016) cita o uso da técnica Think-Aloud Protocols (Protocolos de Pensar em Voz Alta), como um exemplo de avaliação on-line, pois o participante irá verbalizar seus pensamentos em relação à atividade que está realizando. Há ainda, a avaliação por meio do Report Writing (Relatório Escrito), na qual o participante escreve seus comportamentos e o Talking About (Falando Sobre) que, mais do que descrever comportamentos, possibilita uma reflexão sobre itens essenciais do processo de aprendizagem (Panaoura \& Philippou, 2005; Schelini et al., 2016).

Já as avaliações off-line ocorrem anteriormente ou posteriormente à realização de uma atividade; por exemplo, quando o sujeito é requisitado a descrever as estratégias metacognitivas utilizadas na resolução de um problema antes ou após a realização do mesmo, e não durante a execução da tarefa (Desoete, 2008). Levando-se em consideração que as avaliações off-line podem ser realizadas antes ou após a tarefa, é importante despender atenção especial à avaliação efetuada após a atividade: quanto maior o intervalo de tempo entre a conclusão da tarefa e o momento da avaliação, mais a susceptibilidade do indivíduo a distorcer ou sofrer lapsos de memória em relação ao seu desempenho. É provável que sujeitos com baixas habilidades de auto-observação apresentem relatos com menos acurácia de seus reais desempenhos nas tarefas (Boruchovitch, Schelini, \& Santos, 2010; Desoete, 2008; Schelini et al., 2016).

Outra maneira de se avaliar o monitoramento metacognitivo é a partir do julgamento metacognitivo (Cheng, 2010; Efklides, 2006; Nelson \& Narens, 1994; Schelini et al., 2016; Son \& Schwartz, 2002), que se refere ao julgamento probabilístico, uma reflexão do sujeito sobre sua performance (JoPs) antes, durante ou depois de uma tarefa (Fleming, Massoni, Gajdos \& Vergnaud, 2016; Schraw, 2009). Os julgamentos prospectivos são aqueles emitidos antes do início ou durante a tarefa, e são estimativas do indivíduo sobre sua atuação a ser apresentada. Exemplos de julgamentos prospectivos são Ease-of-Learning (Facilidade de Aprendizagem), a estimativa de tempo de estudo para o entendimento de determinada informação; Judgements of Learning (Julgamentos de Aprendizagem - JoLs), a estimativa da probabilidade de que um item ou informação recém-aprendida será lembrado com sucesso; e Feeling-of-Knowing (Sensação de Conhecimento - FoK), o reconhecimento pelo indivíduo de quais informações não foram recordadas (Robey, Dougherty \& Buttaccio, 2017; Schelini et al., 2016). Já os julgamentos retrospectivos, ou de confiança (RCJs), 
são os emitidos após a realização da tarefa, refletindo uma estimativa, descrição ou comentário do sujeito sobre a probabilidade de sucesso em uma tarefa já realizada, ou seja, a probabilidade de alcance do objetivo da tarefa (Huff \& Nietfeld, 2009; Fitzgerald, Arvaneh, \& Dockree, 2017; Son \& Schwartz, 2002; Zampieri \& Schelini, 2013a).

De acordo com Schraw (2009), os julgamentos metacognitivos podem ser realizados de várias maneiras, sendo que a alternativa mais comum é a realização de um continuum de confiança que vai de não confiante para totalmente confiante. Uma segunda possibilidade é realizar uma predição dicotômica de performance bem-sucedida ou malsucedida.

Embora as pessoas sejam consideravelmente precisas em estimar o quão bem aprenderam algo, muitos estudos têm apresentado que os julgamentos metacognitivos dos indivíduos sobre sua memória podem ser mal avaliados. Em muitos julgamentos de aprendizagem (JoLs), indivíduos tipicamente se mostram muito confiantes na nova aprendizagem, sendo o julgamento mais alto que a performance real do teste subsequente (Benjamin, Bjork, \& Schwartz, 1998; Finn, 2008; Koriat, 1997; Koriat, Sheffer, \& Ma'yan, 2002; Metcalfe, 1998; Zechmeister \& Shaughnessy, 1980).

A acurácia de julgamento parece variar de acordo com diferentes fatores, que exercem influência sobre o processo de monitoramento metacognitivo (Kessel et al., 2014). Alguns desses fatores são específicos de memória, como, por exemplo, a familiaridade e a facilidade de processamento dos itens. Resultados indicam que palavras de alta frequência, que são assumidas como mais fluentemente processadas, intensificam os JoLs (Kessel et al., 2014; Koriat, 2007). Além disso, outros fatores relacionados à memória também influenciam, como a dificuldade da tarefa e o tempo de avaliação do julgamento metacognitivo. Estudos que investigaram esses fatores observaram que indivíduos monitoram melhor sua performance depois da realização das tarefas, quando comparadas com a avaliação realizada antes. Ainda, descobriram melhor acurácia de tarefas simples em relação às tarefas mais complexas (Kessel et al., 2014; Schraw, Dunkle, Bendixen, \& Roedel, 1995; Siedlecka, Paylewicz, \& Wierzchoń, 2016).

Vadhan e Stander (1993) realizaram um estudo sobre a habilidade metacognitiva de universitários, pedindo aos participantes que atribuíssem uma nota que acreditavam ter recebido em um exame. Os autores comparam a nota estimada à nota da prova, ou seja, compararam o desempenho real ao desempenho estimado. Os resultados revelaram que os sujeitos que demonstraram julgamento mais acurado, ou seja, com menor diferença entre desempenho previsto e real, alcançaram notas mais altas no teste.

Zampieri e Schelini (2013a) desenvolveram um estudo com o objetivo de explorar o monitoramento metacognitivo de crianças do Ensino Fundamental a partir de três atividades que avaliavam capacidades intelectuais de uma bateria 
de inteligência. Os resultados mostraram que os sujeitos obtiveram escores mais elevados nos julgamentos de confiança vinculados a um subteste que avaliava o raciocínio indutivo e, em referência à precisão do julgamento, observou-se que os participantes foram capazes de emitir estimativas positivas em relação às questões corretas. Num segundo estudo, Zampieri e Schelini (2013b) observaram potenciais relações entre a precisão do monitoramento metacognitivo e o desempenho de estudantes do Ensino Fundamental na mesma bateria de inteligência. A partir dos resultados, verificou-se que os alunos apresentaram maior pontuação no subteste de raciocínio indutivo e alta confiança no acerto de questões em todos os subtestes, mesmo quando apresentaram baixo desempenho.

Schelini et al. (2016) investigaram a avaliação do monitoramento metacognitivo em pesquisas nacionais e internacionais entre 2005 e 2015 . Foram analisados 51 artigos, e os resultados obtidos revelaram que o ano mais produtivo em publicações sobre o monitoramento metacognitivo foi 2012, com 10 publicações, seguido por 2014 (9 publicações). A maioria dos estudos encontrados avaliou o monitoramento metacognitivo por meio de outras técnicas que não testes, escalas, inventários e questionários; e as técnicas mais utilizadas na avaliação do monitoramento foram: Feeling-of-Knowing Task (FoK); ThinkAloud Protocols, Judgements of Learning (JoLs), Metacognitive Awareness Inventory (MAI) e o Met.a.ware. Além disso, os 51 estudos encontrados nos últimos dez anos sobre o monitoramento metacognitivo tiveram amostras compostas principalmente por estudantes universitários, não sendo encontrado qualquer estudo com populações de analfabetos ou em processo de alfabetização, o que revela uma grande carência de pesquisas sobre o tema com a população contemplada no presente estudo (Schelini et al., 2016).

\section{Alfabetização e letramento}

Apesar de serem conceitos que muitas vezes se interpõem, a alfabetização e o letramento se distinguem, principalmente, em sua aplicabilidade para o indivíduo. A alfabetização é um processo interpretativo (Haddad \& Siqueira, 2015), um conjunto de habilidades relacionadas à aprendizagem da leitura e escrita; e, até 1958, a UNESCO estabelecia que o sujeito alfabetizado era aquele capaz de ler e escrever discursos simples e pertencentes ao dia-a-dia (Ribeiro, 2006). Já o letramento, termo concebido em meados da década de 1980 no Brasil, é utilizado como forma de se diferenciar as habilidades de leitura e escrita mais desenvolvidas que os exercícios de ler e escrever consequentes do conhecimento do sistema de escrita; é uma evolução no uso do ler e escrever em âmbito social, pelo qual o indivíduo se integra ativa e competentemente com o ambiente ao redor, expandindo suas capacidades de prática de leitura e escrita em atividades que compreendem a língua escrita (Moraes, 2005; Soares, 2004). 
De acordo com o Ministério da Educação, o sujeito alfabetizado é aquele que possui domínio da escrita, leitura e conceitos numéricos (Presidência da República, 2014). Possui, também, habilidade de usar tais conhecimentos em âmbito social, sendo capaz de utilizar tais competências de diversas maneiras (Ribeiro, 2006).

O presente estudo utilizou quatro possíveis classificações para a condição de alfabetismo (Ribeiro, Vóvio \& Moura, 2002): 1) analfabetismo; 2) alfabetismo nível 1 (indivíduo que possui capacidades rudimentares de alfabetização e letramento); 3) alfabetismo nível 2 (indivíduo com compreensão mínima de alfabetização e aplicação de suas capacidades em ambiente social); e 4) alfabetismo nível 3 (indivíduo possui habilidades de alfabetização e letramento que constituem o mínimo estabelecido pela educação básica. No entanto, ressalva-se que não há limites para o letramento, tendo em vista que a própria evolução social se dá por novas configurações linguísticas cada vez mais avançadas. Há, no entanto, graus de letramento, um continuum, que, apesar de não absolutos ou definitivos, auxiliam na classificação das habilidades dos participantes.

O Brasil possui 14 milhões de analfabetos, sendo a oitava nação com a maior população de analfabetos no mundo (UNESCO, 2014). O interesse em explorar o monitoramento metacognitivo em adultos analfabetos e em processo de alfabetização ocorreu pela escassez de estudos com tal população. A metacognição é instrumento essencial para a aprendizagem formal, podendo ser impulsionado pela educação (Cornoldi, 2010; Jou \& Sperb, 2006).

Fujie e Schelini (2018) investigaram o monitoramento metacognitivo de adultos participantes de um programa de alfabetização em atividades que avaliavam o fator geral de inteligência, velocidade de processamento e memória de curto prazo a partir do julgamento de seu desempenho. Além disso, foram observadas as diferenças entre desempenhos reais e estimados e quais atividades o desempenho real mais se relacionava ao desempenho estimado. Ao final da aplicação do Teste R-1 e subtestes Código, Procurar Símbolos e Dígitos da Escala Wechsler de Inteligência para Adultos III (WAIS-III), era apresentado o Registro de Julgamentos, pelo qual o participante realizava um julgamento retrospectivo, dando um valor de 0 a 100 a seu desempenho. Os resultados evidenciaram que os participantes obtiveram escores totais brutos e percentis abaixo da média; tenderam a qualificar seu próprio desempenho como baixo; e a análise da correlação entre o escore bruto do desempenho real e julgamento de desempenho exibiu níveis de correlação de baixa magnitude, sendo significativa apenas a correlação entre o subteste Códigos e seu julgamento.

Não foram encontrados outros estudos acerca do monitoramento metacognitivo em adultos analfabetos ou em processo de alfabetização, após levantamento nacional e internacional nas plataformas SciELo, Pepsic, LivRe e Index Psi, sem especificação de período de tempo, sendo o mesmo observado no 
trabalho de Schelini et al. (2016), após análise nas plataformas PsycInfo, Web of Science e SciELo, entre os anos de 2005 e 2015.

0 presente estudo teve por objetivo investigar 0 monitoramento metacognitivo em adultos num programa de alfabetização por meio do julgamento de desempenho em tarefas cognitivas e possíveis correlações entre desempenho real e estimado.

\section{MÉTODO}

\section{Participantes}

Participaram do estudo 15 adultos, 12 do gênero feminino e 3 do gênero masculino, com idades entre 20 e 74 anos (média de 44,87 anos), sendo 10 analfabetos e 5 alfabetizados nível 1 .

\section{Instrumentos}

\section{Entrevista Inicial}

Empregada para a obtenção de dados de identificação e escolaridade dos participantes.

Teste $R-1$ : Teste Não Verbal de Inteligência

Criado por Oliveira (1973) e adaptado por Alves (2002), com o objetivo de aferir a inteligência de adultos, notadamente o fator geral. Possui 40 itens não verbais, exibidos em um caderno, com um item diferente por página, de forma a evitar interferência de um item sobre o outro, possibilitando a maior concentração do examinado. Os itens são compostos por figuras incompletas, e que devem ser concluídas por uma das alternativas exibidas abaixo das mesmas.

Ao longo do teste, a complexidade dos itens aumenta com o uso de figuras geométricas e compreensão de relações de identidade e semelhança, além do acréscimo da coleção de elementos na matriz e relações de soma e subtração (Gottsfritz \& Alves, 2009). As respostas são assinaladas em uma folha de respostas, possibilitando uma aplicação individual ou coletiva. O R-1 é um teste com estudos que demonstram sua validade e precisão para uso na população brasileira. No presente estudo, o instrumento foi aplicado individualmente.

Escala Wechsler de Inteligência para Adultos III (WAIS-III)

Elaborada por Wechsler (1997) e adaptada para uso no Brasil por Nascimento (2005), foi designada a fim de investigar o desempenho intelectual de adolescentes e adultos. É constituída por 14 subtestes, reunidos em dois conjuntos: Verbal (subtestes Aritmética, Compreensão, Dígitos, Informação, Semelhanças, Sequência de Números e Letras, e Vocabulário) e Execução (subtestes Arranjo de Figuras, Armar Objetos, Códigos, Completar Figuras, Cubos, Procurar Símbolos e Raciocínio Matricial). Foram selecionados para a 
presente pesquisa os subtestes Código e o Procurar Símbolos, que avaliam a velocidade de processamento cognitivo, e Dígitos, que avalia a memória de curto prazo.

\section{Registro de julgamentos}

Composto por uma folha de registro elaborada com base no material desenvolvido por Zampieri (2012), com o propósito do participante aferir seu desempenho no Teste R-1 e nos subtestes Código, Procurar Símbolos e Dígitos do WAIS-III. Ao fim de cada atividade, a pesquisadora lia a seguinte instrução ao participante: "Pense no teste que acabou de fazer. Indique de 0 a 10, qual você acha que é a chance de ter acertado este teste, como se fosse uma nota para o que fez", de forma que o indivíduo desempenhava, então, um julgamento retrospectivo da sua performance. Ainda, o participante recebia seu número máximo de suas respostas em cada atividade e, a partir desse valor, era exibida uma régua métrica e informada a seguinte instrução: "Você respondeu/completou $X$ questões/linhas. Olhe para a régua à sua frente, que contém o número máximo de questões/linhas que você finalizou. Dessas $X$ respostas, quantas você acha que acertou?". Se o participante havia, por exemplo, completado 20 linhas do subteste Procurar Símbolos, a pesquisadora apontava na régua o intervalo de 0 a 20 e dava a instrução para que o participante fizesse um julgamento retrospectivo de seu desempenho.

\section{Procedimentos}

O projeto foi aprovado pelo Comitê de Ética em Pesquisas em Seres Humanos (CAAE 48461015.3.0000.5504). Somente participaram da pesquisa sujeitos que acordaram com o Termo de Consentimento Livre e Esclarecido (TCLE), que compreendia informações e explicações sobre o procedimento e objetivos do estudo.

O primeiro contato da pesquisadora com os possíveis participantes foi feito com o intuito de explicar o objetivo da pesquisa e constatar o interesse em participação. Posteriormente, foi ajustado um horário de encontro para a coleta de dados. Cada encontro individual durou cerca de 60 minutos, e a sessão foi subdivida da seguinte forma: 1) Esclarecimento da pesquisa, leitura do TCLE e aplicação da Entrevista Inicial; 2) aplicação do Teste R-1 e Registro de Julgamentos; 3) aplicação dos subtestes Código e Registro de Julgamentos; Procurar Símbolos e Registro de Julgamentos; e Dígitos e Registro de Julgamentos.

\section{Análise de dados}

Os dados da Entrevista Inicial foram sistematizados em roteiro, de forma a propiciar sua avaliação; e na análise do monitoramento metacognitivo, os escores brutos dos participantes no Teste R-1 e subtestes Códigos, Procurar 
Símbolos e Dígitos foram correlacionados aos desempenhos estimados pelos participantes. Adicionalmente, foram correlacionados os escores ponderados e os julgamentos dos participantes.

\section{RESULTADOS}

De acordo com a estatística descritiva sobre o desempenho dos participantes no Teste R-1 e subtestes Códigos, Dígitos e Procurar Símbolos da Escala Wechsler de Inteligência para Adultos III (WAIS-III), o teste que apresentou a menor média de pontuação foi o subteste Procurar Símbolos $(8,80)$, também tendo o menor desempenho mínimo. Já o teste com a maior média de pontuação foi o subteste Códigos $(19,67)$, seguido pelo Teste R-1. Essas médias devem ser interpretadas com discernimento, uma vez que as pontuações máximas possíveis variam de teste para teste.

No julgamento estimado de 0 a 10, constata-se que todos os testes tiveram participantes que julgaram seu desempenho com nota mínima 0 , assim como participantes que julgaram seu desempenho com nota máxima 10. Além disso, o subteste Procurar Símbolos teve a maior média de julgamento de desempenho $(7,53)$, seguido pelo Teste $\mathrm{R}-1$.

Já no desempenho estimado por meio de um objeto de medida (régua), observou-se que o subteste Dígitos apresentou a menor nota mínima, assim como a menor média de nota de julgamento $(9,07)$. O Teste R-1 e o subteste Procurar Símbolos apresentaram as maiores notas mínimas e as maiores notas máximas e o Teste R-1 apresentou a maior média de nota de julgamento $(16,87)$.

Uma vez indicadas as informações a respeito do desempenho dos participantes no Teste R-1 e subtestes Códigos, Dígitos e Procurar Símbolos, serão apresentadas a seguir a classificação de resultados a partir dos seus percentis (Tabela 1 e Tabela 2).

Tabela 1.

Percentis por Amostra Total no Teste R-1

\begin{tabular}{ccc}
\hline Classificação (percentis) & $\mathrm{N}$ & $\%$ \\
\hline Inferior $(1-5)$ & 9 & 60,00 \\
Médio Inferior $(10-25)$ & 5 & 33,33 \\
Médio $(30-70)$ & 1 & 6,70 \\
Médio Superior $(75-90)$ & 0 &, 00 \\
Superior (95) & 0 &, 00 \\
Muito Superior (>99) & 0 &, 00 \\
\hline
\end{tabular}


Nota-se que a maioria dos participantes apresentou desempenho inferior $(60 \%)$ ou médio inferior (33,33\%) no Teste R-1, com nenhum participante atingindo a classificação média superior, superior ou muito superior.

Tabela 2.

Percentis por Amostra Total nos subtestes Códigos, Dígitos e Procurar Símbolos

\begin{tabular}{ccccccc}
\hline $\begin{array}{c}\text { Classificação } \\
\text { (percentis) }\end{array}$ & Códigos (n) & $\%$ & $\begin{array}{c}\text { Dígitos } \\
(\mathrm{n})\end{array}$ & $\%$ & $\begin{array}{c}\text { Procurar } \\
\text { Símbolos } \\
(\mathrm{n})\end{array}$ & $\%$ \\
\hline $1-5$ & 4 & 26,60 & 1 & 6,7 & 3 & 20,00 \\
$6-10$ & 11 & 73,40 & 11 & 73,3 & 12 & 80,00 \\
$11-15$ & 0 &, 00 & 3 & 20,00 & 0 &, 00 \\
$16-19$ & 0 &, 00 & 0 &, 00 & 0 &, 00 \\
\hline
\end{tabular}

Na classificação dos participantes pelos percentis nos subtestes Códigos, Dígitos e Procurar Símbolos, nenhum participante obteve os maiores valores, sendo que a maior parte dos desempenhos se concentrou entre os percentis $6 \mathrm{e}$ 10.

Utilizando-se os valores de assimetria entre -3 e +4 (Kline, 2011) e valores de curtose entre -8 e +8 (Kline, 2011) para uma distribuição normal, pode-se observar que, em relação ao Teste $\mathrm{R}-1$, as respostas dos 15 participantes apresentaram uma distribuição que tendeu à normalidade (Assimetria $=0,720$; Curtose $=0,841$ ), assim como nos subtestes Códigos (Assimetria $=1,279 ;$ Curtose $=1,525$ ), Dígitos (Assimetria $=0,115$; Curtose = 1,289) e Procurar Símbolos (Assimetria $=0,334$; Curtose $=0,265$ ). Dessa maneira, foram utilizados testes estatísticos paramétricos para realizar análises correlacionais entre os escores brutos e julgamentos de desempenho e entre os percentis e julgamentos de desempenho.

A seguir, apresentam-se as correlações de Pearson entre os escores totais brutos e os julgamentos de desempenho a partir de um objeto de medida dos participantes no Teste R-1 e subtestes Códigos, Dígitos e Procurar Símbolos (Tabela 3).

Tabela 3.

Correlações de Pearson entre os escores brutos do Teste R-1 e os subtestes Códigos, Dígitos e Procurar Símbolos e seus respectivos julgamentos a partir de um objeto de medida

R-1 Códigos Dígitos Procurar Símbolos

\begin{tabular}{ccc}
\hline Julgamento R-1 &,- 330 & \\
Julgamento Códigos &, $741^{* *}$ & \\
Julgamento Dígitos & & $0,576^{*}$
\end{tabular}


* Correlação de Pearson significativa a 0,05

** Correlação de Pearson significativa a 0,01

Considerando as correlações estabelecidas entre os escores brutos do Teste R-1 e os subtestes Códigos, Dígitos e Procurar Símbolos com os julgamentos dos participantes em relação ao seu desempenho em cada atividade, pode-se observar que houve correlação alta e significativa, entre 0 subteste Códigos e os julgamentos $(r=0,741)$, alta e significativa entre 0 subteste Dígitos e os julgamentos $(r=0,576)$ e alta e significativa entre subteste Procurar Símbolos e os julgamentos $(r=0,703)$. Os níveis de associação utilizados foram: alta $r>0,50$, moderada $r=0,35-0.50$ e fraca $r \leq 0,34$ (Dancey \& Reidy, 2013).

A Tabela 4 apresenta a classificação dos julgamentos de desempenho a partir de um objeto de medida dos participantes em relação número de itens respondidos.

Tabela 4.

Classificação do julgamento de desempenho a partir de um objeto de medida dos participantes em relação ao número de itens respondidos

\begin{tabular}{ccccc}
\hline & $\mathrm{N}$ & Subestimou (\%) & Superestimou (\%) & Acertou (\%) \\
\hline R-1 & 15 & 46,70 & 46,70 & 6,60 \\
Códigos & 15 & 66,70 & 6,70 & 26,60 \\
Dígitos & 15 & 73,30 & 20,00 & 6,70 \\
Símbolos & 15 & 33,30 & 66,70 & - \\
\hline
\end{tabular}

De acordo com o julgamento do participante, era possível classificar sua resposta como subestimando, superestimando ou estimando corretamente seu real desempenho. Pode-se observar que o subteste Dígitos teve o maior número de participantes subestimando seu desempenho $(73,3 \%)$, seguido pelo subteste Códigos. O subteste Procurar Símbolos foi o único teste que apresentou a maioria dos participantes como superestimando seu desempenho $(66,7 \%)$, sendo que em nenhuma das atividades a maioria dos participantes estimou corretamente seu desempenho real.

A Tabela 5 apresenta as correlações de Pearson entre os escores brutos do Teste R-1 e subtestes Códigos, Dígitos e Procurar Símbolos com o julgamento dos participantes sobre seu desempenho de 0 a 10. 
Tabela 5.

Correlações de Pearson entre os escores brutos do Teste R-1 e os subtestes Códigos, Dígitos e Procurar Símbolos e seus respectivos julgamentos de 0 a 10

\begin{tabular}{|c|c|c|c|c|}
\hline & $\mathrm{R}-1$ & Códigos & Dígitos & Procurar Símbolos \\
\hline Julgamento R-1 &,- 026 & & & \\
\hline Julgamento Códigos & &,- 079 & & \\
\hline Julgamento Dígitos & & & 0,400 & \\
\hline $\begin{array}{c}\text { Julgamento Procurar } \\
\text { Símbolos }\end{array}$ & & & & 0,190 \\
\hline
\end{tabular}

Pode-se observar que as correlações estabelecidas entre os escores brutos das atividades com os julgamentos dos participantes em relação a seu desempenho a partir do estabelecimento de nota de 0 a 10 foram de fraca magnitude e não significativas. A exceção ocorreu entre o subteste Dígitos e seu julgamento, cuja correlação pode ser considerada moderada, de acordo com Dancey e Reidy (2013).

A Tabela 6 apresenta as correlações de Pearson entre os percentis dos participantes no Teste R-1 e subtestes Códigos, Dígitos e Procurar Símbolos com seus julgamentos de desempenho a partir de um objeto de medida.

Tabela 6.

Correlações de Pearson entre os percentis do Teste R-1 e os subtestes Códigos, Dígitos e Procurar Símbolos e seus respectivos julgamentos a partir de um objeto de medida

\begin{tabular}{|c|c|c|c|c|}
\hline & $\mathrm{R}-1$ & Códigos & Dígitos & Procurar Símbolos \\
\hline Julgamento R-1 &,- 108 & & & \\
\hline Julgamento Códigos & & ,267 & & \\
\hline Julgamento Dígitos & & & $0,641 *$ & \\
\hline $\begin{array}{c}\text { Julgamento Procurar } \\
\text { Símbolos }\end{array}$ & & & & 0,316 \\
\hline
\end{tabular}

* Correlação de Pearson significativa a 0,05

É possível observar que houve correlação significativa e de alta magnitude apenas entre os percentis do subteste Dígitos e seus julgamentos $(r=0,641)$.

A Tabela 7 apresenta as correlações de Pearson entre os percentis dos participantes no Teste R-1 e subtestes Códigos, Dígitos e Procurar Símbolos com seus julgamentos de desempenho de 0 a 10 nos mesmos. 
Tabela 7.

Correlações de Pearson entre os percentis do Teste R-1 e os subtestes Códigos, Dígitos e Procurar Símbolos e seus respectivos julgamentos de 0 a 10

\begin{tabular}{ccccc}
\hline & $\mathrm{R}-1$ & Códigos & Dígitos & Procurar Símbolos \\
\hline Julgamento R-1 &, 034 & & & \\
Julgamento Códigos & &,- 318 & & \\
Julgamento Dígitos & & &, 330 & \\
$\begin{array}{c}\text { Julgamento Procurar } \\
\text { Símbolos }\end{array}$ & & &,- 063 \\
\hline
\end{tabular}

É possível observar que não houve correlações significativas entre os percentis e os julgamentos de desempenho de 0 a 10 dos participantes.

\section{DISCUSSÃO}

Assim como no estudo de Fujie e Schelini (2018), o objetivo deste estudo foi investigar o monitoramento metacognitivo de adultos em processo de alfabetização utilizando-se o julgamento (estimativa) sobre o desempenho em tarefas cognitivas. Foram realizadas análises entre os escores brutos, percentis e julgamentos, de maneira a correlacionar os desempenhos reais e estimados dos participantes, para se verificar a existência de correlações significativas entre os resultados.

É possível observar que os escores totais brutos e percentis obtidos pelos participantes no Teste R-1 e subtestes Códigos, Dígitos e Procurar Símbolos foram baixos. Similarmente aos resultados encontrados de Fujie e Schelini (2018), este fato parece ter sido comprovado em diversos outros estudos, apontando a existência de diferenças significativas entre os escores de grupos com diferentes níveis de escolaridade em atividades de memória de curto prazo, velocidade de processamento, assim como o desempenho no Teste R-1 (Alves, 1998; Banhato \& Nascimento, 2007; Parente, Saboskinski, Ferreira, \& Nespoulous, 1999; Tommasi et al., 2015).

O presente estudo utilizou duas formas de registro de julgamento de desempenho. Em uma delas, o participante recebia o número total de suas questões respondidas e observava o intervalo entre zero e o valor máximo de suas respostas numa régua. Após essa observação, ele realizava o julgamento de seu desempenho.

A análise da correlação de Pearson entre os escores brutos do desempenho real e o julgamento de desempenho a partir de um objeto (régua) de medida apresentou níveis de correlação significativos e de alta magnitude nos subtestes Códigos e Procurar Símbolos, e níveis de correlação significativos e de média magnitude no subteste Dígitos. Uma possível explicação para esse 
resultado é a maior facilidade dos subtestes Códigos e Procurar Símbolos em relação às outras atividades, sendo ambas tarefas de baixa complexidade e que têm a velocidade de processamento como um fator decisivo.

A partir do julgamento de desempenho a partir de um objeto de medida, foi possível classificar as respostas dos participantes entre subestimação, superestimação ou estimação correta de seu desempenho real. Os resultados apresentados indicam que no Teste R-1 e subtestes Códigos e Dígitos, os participantes subestimaram seu desempenho, observando-se o contrário no subteste Procurar Símbolos. O fato dos participantes terem subestimado seus desempenhos mesmo em tarefas mais fáceis, como o subteste Códigos, parece estar relacionado à condição de que a escolaridade afeta o nível de autoestima (Santos, Eulálio, Melo, \& Nunes, 2014; Siqueira, 2010), o que levaria os sujeitos a avaliarem seu desempenho como pior que seu desempenho real. Já a superestimação de desempenho no subteste Procurar Símbolos pode estar relacionada ao fato deste subteste ser a atividade de maior facilidade dentre todas as atividades, o que levaria o participante a avaliar seu desempenho como melhor que seu desempenho real. A partir destes resultados, é possível observar que o novo método de analisar o julgamento metacognitivo proporcionou relações mais fortes e, possivelmente, mais acuradas do que o método utilizado no estudo de Fujie e Schelini (2018)

A outra forma de julgamento utilizada foi a partir da estimativa de desempenho entre zero e dez. Diferentemente do estudo de Fujie e Schelini (2018), que utilizava o intervalo de zero a cem, este julgamento solicitava ao participante que desse uma nota entre zero e dez para o seu desempenho em cada atividade.

A análise de correlação de Pearson entre o escore bruto do desempenho real e seu julgamento de desempenho entre zero e dez não apresentou quaisquer correlações significativas, sendo todos os níveis de correlação de baixa magnitude. Aparentemente, o uso de um intervalo maior de valores facilita o julgamento de desempenho dos participantes, uma vez que no estudo de Fujie e Schelini (2018) foram observados níveis de correlação positivos e de baixa magnitude, mas significativo no subteste Códigos e seus julgamentos. Uma possível razão para isso talvez seja que quanto maior o intervalo numérico de julgamento, mais fácil seja para o participante visualizar, categorizar e numerar seu desempenho.

A correlação entre os percentis e ambos os julgamentos de desempenho só foi significativa e de moderada magnitude entre o subteste Dígitos e o julgamento a partir de um objeto de medida. Esse resultado parece sugerir ser mais adequado utilizar escores brutos e não os Percentis em futuras análises de dados, uma vez que os participantes foram estimulados a indicar um valor de acerto a partir de um total de itens realizados, e não a indicar qual seria sua 
posição quando comparados a outras pessoas (100 pessoas), sendo este o pressuposto do Percentil.

Fatores como a dificuldade da tarefa e o tempo entre a realização da tarefa e o julgamento de desempenho afetam a acurácia do julgamento dos participantes (Kessel et al., 2014; Schraw, Dunkle, Bendixen, \& Roedel, 1995). A correlação significativa entre os escores brutos e os julgamentos a partir de um objeto de medida dos subtestes Códigos, Dígitos e Procurar Símbolos, em relação à correlação negativa e de baixa magnitude do Teste $R-1$ e seu julgamento, pode, então, estar fundamentada na maior facilidade dos subtestes em relação ao Teste R-1. Ainda, assim como no estudo de Fujie e Schelini (2018), esses dados sugerem que um melhor desempenho nas atividades está, em geral, relacionado a um melhor monitoramento metacognitivo (Son \& Schwartz, 2005; Vadhan \& Stander, 1993; Zampieri \& Schelini, 2013b).

\section{CONSIDERAÇÕES FINAIS}

O campo da metacognição e, mais especificamente, do monitoramento metacognitivo, ainda carece de estudos. É um tema pouco investigado, com necessidade de maior exploração e desenvolvimento de conhecimento. Ainda, não existem muitas pesquisas sobre a população aqui escolhida, dificultando sua investigação.

Juntamente com o estudo de Fujie e Schelini (2018), o presente estudo foi um dos poucos realizados no Brasil com o objetivo de investigar 0 monitoramento metacognitivo em adultos em processo de escolarização. Seu desenvolvimento ocorreu de forma a tentar minimizar as lacunas na investigação de Fujie e Schelini (2018), como a falta de coincidência entre a pontuação total dos testes aplicados e o registro de julgamentos; e os resultados obtidos parecem indicar um progresso em relação ao melhor método a ser empregado para a realização de julgamentos retrospectivos. Sugere-se, também, a realização de novos estudos sobre o tema e com a mesma população, com um número maior de participantes e diferentes tipos e quantidade de testes aplicados.

O baixo desempenho dos participantes em relação à média da população demonstra o impacto da falta de educação formal ao desenvolvimento cognitivo. Isso indica a necessidade de investimento às políticas públicas de educação, de forma a ampliar e reformar as instituições de ensino e a inclusão de adultos em programas de alfabetização.

\section{DECLARAÇÃO DE CONFLITO DE INTERESSES}

As autoras declaram que não há conflito de interesses. 


\section{REFERÊNCIAS}

Alves, I. C. B. (2002). R-1: Teste Não Verbal de Inteligência - Manual. São Paulo, SP: Vetor.

Alves, I. C. B. (1998). Variáveis significativas na avaliação da inteligência. Psicologia Escolar e Educacional, 2(2), 109-114. doi:10.1590/S141385571998000200005

Banhato, E. F. C., \& Nascimento, E. D. (2007). Função executiva em idosos: Um estudo utilizando subtestes da Escala WAIS-III. Psico-USF, 12(1), 6573. doi: $10.1590 / S 1413-82712007000100008$

Benjamin, A. S., Bjork, R. A., \& Schwartz, B. L. (1998). The mis-measure of memory: When retrieval fluency is misleading as a meta-mnemonic index. Journal of Experimental Psychology: General, 127, 55-68. doi: 10.1037/0096-3445.127.1.55

Boruchovitch, E., Schelini, P. W., \& Santos, A. A. A. (2010). Metacognição: Conceituação e medidas. In A. A. A. Santos, F. F. Sisto, E. Boruchovitch, \& Nascimento, E. (Eds.), Perspectivas em Avaliação Psicológica (pp. 123-143). São Paulo, SP: Casa do Psicólogo.

Brown, A. L. (1978). Knowing when, where, and how to remember: A problem of metacognition. In R. Glases (Ed.), Advances in Instructional Psychology (77-165). Hillside, NJ: Lawrence Erlbaum Associates.

Cheng, C. M. (2010). Accuracy and stability of metacognitive monitoring: A new measure. Behavior Research Methods, 42(3), 715-732. doi: $10.3758 / B R M .42 .3 .715$

Cornoldi, C. (2010). Metacognition, intelligence, and academic performance. In H. S. Waters, \& W. Schneider (Eds.), Metacognition, Strategy Use, and Instructions (pp. 257-277). New York, NY: The Guilford Press.

Dal'Evedove, P. R., Neves, D. A. D. B., \& Fujita, M. S. L. (2014). A metacognição de usuários no processo de busca da informação em catálogo coletivo de biblioteca universitária. Perspectivas em Ciência da Informação, 25-42. doi:10.1590/1981-5344/1794

Dancey, C. P., \& Reidy, J. (2013). Estatística sem Matemática para Psicologia. Porto Alegre, RS: Artmed.

Desoete, A. (2008). Multi-method assessment of metacognitive skills in elementary school children: What you test is what you get. Metacognition Learning, 3, 189-206. doi:10.1007/s11409-008-9026-0

Efklides, A. (2006). Metacognition and affect: What can metacognitive experiences tell us about the learning process? Educational Research Review, 1(1), 3-14. doi:10.1016/j.edurev.2005.11.001

Finn, B. (2008). Framing effects on metacognitive monitoring and control. Memory \& Cognition, 36(4), 813-821. doi:10.3758/MC.36.4.813 
Fitzgerald, L. M., Arvaneh, M., \& Dockree, P. M. (2017). Domain-specific and domain-general processes underlying metacognitive judgments. Consciousness and Cognition, 49, 264-277. doi: $10.1016 /$ j.concog.2017.01.011

Flavell, J. H., \& Wellman, H. M. (1977). Metamemory. In R. V. Kail, \& J. W. Hagen (Eds.), Perspectives on the Development of Memory and Cognition (pp. 3-33). Hillsdale, NJ: Lawrence Erlbaum Associates.

Flavell, J. H. (1987). Speculations about the nature and development of metacognition. In F. E. Weinert, \& R. H. Kluwe (Eds.), Metacognition, motivation and understanding (pp. 21-29). Hillsdale, NJ: Lawrence Erlbaum Associates.

Fleming, S. M., Massoni, S., Gajdos, T., \& Vergnaud, J-C. (2016). Metacognition about the past and future: Quantifying common and distinct influences on prospective and retrospective judgments of self-performance. Neuroscience of Consciouness, 2016(1), 1-12. doi:10.1093/nc/niw018

França, A. B., \& Schelini, P. W. (2018). Escala de avaliação da metacognição em idosos: Evidências de validade e consistencia interna. Psicologia: Teoria e Pesquisa, 33. doi:10.1590/0102.3772e3324

Fujie, M. A., \& Schelini, P. W. (2018). Monitoramento metacognitivo de adultos em processo de alfabetização em tarefas de memória, processamento e inteligência geral. Psico, 49(3), 285-293. doi:10.15448/19808623.2018.3.27955

Gotsfritz, M. O., \& Alves, I. C. B. (2009). Normas do teste de inteligência não verbal R-1 para adultos não alfabetizados. Interação em Psicologia, 13(1), 59-68. doi:10.5380/psi.v13i1.15856

Haddad, S., \& Siqueira, F. (2015). Analfabetismo entre jovens e adultos no Brasil. Revista Brasileira de Alfabetização - ABAlf, 1(2), 88-110.

Huff, J.D., \& Nietfeld, J.L. (2009). Using strategy instruction and confidence judgements to improve metacognitive monitoring. Metacognition and Learning, 4(2), 161-176. doi:10.1007/s11409-009-9042-8

Jou, G. I., \& Sperb, T.M. (2006). A metacognição como estratégia reguladora da aprendizagem. Psicologia: Reflexão e Crítica, 19(2), 177-185. doi: $10.1590 / \mathrm{s} 0102-79722006000200003$

Kessel, R., Gecht, J., Forkmann, T., Drueke, B., Gauggel, S., \& Mainz, V. (2014). Metacognitive monitoring of attention performance and its influencing factors. Psychological Research, 78(4), 597-607. doi: 10.1007/s00426-013-0511-y

Kline, R. B. (2011). Principles and practice of structural equation modeling (3rd ed.). New York, NY: The Guilford Press.

Koriat, A. (1997). Monitoring one's own knowledge during study: A cueutilization approach to judgments of learning. Journal of Experimental Psychology: General, 126(4), 349-370. doi:10.1037/0096-3445.126.4.349 
Koriat, A. (2007). Metacognition and consciousness. In P. D. Zelazo, M. Moscovitch, \& E. Thompson (Eds.), The Cambridge Handbook of Consciousness (pp. 289-325). Cambridge, Inglaterra: Cambridge University Press.

Koriat, A., Sheffer, L., \& Ma'yan, H. (2002). Comparing objective and subjective learning curves: Judgments of learning exhibit increased underconfidence with practice. Journal of Experimental Psychology: General, 131(2), 147-162. doi:10.1037/0096-3445.131.2.147

Lima Filho, R., \& Bruni, A. (2015). Metacognição estimula características empreendedoras? Uma análise em profissionais de administração. RACE Revista de Administração, Contabilidade e Economia, 14(2), 427-450. doi: $10.18593 /$ race.v14i2.5922

Metcalfe, J. (1998). Cognitive optimism: Self-deception or memory-based processing heuristics? Personality \& Social Psychology Review, 2, 100-110. doi: $10.1207 /$ s15327957pspr0202_3

Moraes, M. G. (2005). Alfabetização - leitura do mundo, leitura da palavra - e letramento: Algumas aproximações. Revista de Ciências Humanas, Universidade Regional Integrada do Alto Uruguai e das Missões, 7(7), 1-11.

Nascimento, E. (2005). WAIS-III: Escala de Inteligência Wechsler para Adultos: Manual técnico. São Paulo, SP: Casa do Psicólogo.

Nelson, T. O., \& Narens, L. (1994). Why investigate metacognition. In J. Metcalfe, \& A. P. Shimamura (Eds.), Metacognition: Knowing About Knowing (pp. 1-25). Cambridge, MA: MIT Press.

Oliveira, R. (1973). R-1: Teste Não Verbal de Inteligência - Manual. São Paulo, SP: Vetor.

Organização das Nações Unidas para a Educação, a Ciência e a Cultura. (2014). Ensinar e aprender: Alcançar a qualidade para todos. Paris, França: UNESCO.

Panaoura, A., \& Philippou. G. (2005). The measurement of young pupils' metacognitive ability in mathematics: The case of self-representation and self-evaluation. Journal Proceedings of CERME, 4, 1-10.

Parente, M. A. D. M. P., Saboskinski, A. P., Ferreira, E., \& Nespoulous, J. L. (1999). Memória e compreensão da linguagem no envelhecimento. Estudos Interdisciplinares sobre o Envelhecimento, 1, 57-76.

Presidência da República. Casa Civil. Subchefia para Assuntos Jurídicos (2014). Lei no 13.005, de 25 de junho de 2014. Aprova o Plano Nacional de Educação - PNE e dá outras providências. Diário Oficial da União. Brasília, DF: Câmara dos Deputados.

Reynolds, R., \& Wade, S. (1986). Thinking about thinking about thinking: Reflections on metacognition. Harvard Educational Review, 56(3), 307-318. doi: 10.17763/haer.56.3.a2v45g440pg45173 
Ribeiro, V. M. (2006, julho/agosto). Analfabetismo e alfabetismo funcional no Brasil. Boletim INAF, pp. 6-8. Recuperado de https://www.researchgate.net/publication/265743329_Analfabetismo_e_alf abetismo_funcional_no_Brasil

Ribeiro, V. M., Vóvio, C. L., \& Moura, M. P. (2002). Letramento no Brasil: Alguns resultados do indicador nacional de alfabetismo funcional. Educação \& Sociedade, 23(81), 49-70. doi:10.1590/S0101-73302002008100004

Santos, K. L., Eulálio, M. C., Melo, R. L. P., \& Nunes, R. P. (2014). Autoestima, bem-estar subjetivo e autoavaliação da saúde de pessoas idosas. $10^{\circ}$ Congresso Nacional de Psicologia da Saúde, Sociedade Portuguesa de Psicologia da Saúde. Porto, Portugal.

Schelini, P. W., Deffendi, L. T., Fujie, M. A., Boruchovitch, E., \& Freitas, M. F. R. L. (2016). Avaliação do monitoramento metacognitivo: Análise da produção científica. Avaliação Psicológica, 15(spe), 57-65. doi: 10.15689/ap.2016.15ee.06

Schraw, G. (2009). A conceptual analysis of five measures of metacognitive monitoring. Metacognition Learning, 4, 33-45. doi:10.1007/s11409-0089031-3

Schraw, G., Dunkle, M. E., Bendixen, L. D., \& Roedel, T. D. (1995). Does a general monitoring skill exist? Journal of Educational Psychology, 87(3), 433-444. doi: 10.1037/0022-0663.87.3.433

Siedlecka, M., Paulewicz, B., \& Wierzchoń, M. (2016). But I was so sure! Metacognitive judgments are less accurate given prospectively than retrospectively. Frontiers in Psychology, 7, 218. doi: $10.3389 /$ fpsyg.2016.00218

Siqueira, N. F. (2010). Avaliando a autoestima de adolescentes com epilepsia. (Dissertação de Mestrado). Universidade Estadual de Campinas, São Paulo.

Soares, M. (2004). Letramento e alfabetização: As muitas facetas. Revista Brasileira de Educação, 25, 5-17. doi:10.1590/S1413-24782004000100002

Son, L. K., \& Schwartz, B. L. (2002). The relation between metacognitive monitoring and control. In T. J. Perfect \& B. L. Schwartz (Eds.), Applied Metacognition (pp. 15-35). Cambridge, Inglaterra: Cambridge University Press.

Tanikawa, H. A. M., \& Boruchovitch, E. (2016). Metacognitive Monitoringin students of elementary school. Psicologia Escolar e Educacional, 20(3), 457464. doi: $10.1590 / 2175-3539201502031012$

Tommasi, M., Pezzuti, L., Colom, R., Abad, F. J., Saggino, A., \& Orsini, A. (2015). Increased educational level is related with higher IQ scores but lower g-variance: Evidence from the standardization of the WAIS-R for Italy. Intelligence, 50,68-74. doi:10.1016/j.intell.2015.02.005 
Vadhan, V., \& Stander, P. (1993). Metacognitive ability and test performance among college students. The Journal of Psychology, 128(3), 307-309. doi: 10.1080/00223980.1994.9712733

Wechsler, D. (1997). Wechsler Adult Intelligence Scale - Third Edition (WAISIII). Admnistrations and Scoring Manual. San Antonio, TX: Psychological Corporation.

Weinert, F. E., \& Kluwe, R. H. (1987). Metacognition, Motivation, and Understanding. Hillsdale, NJ: Lawrence Erlbaum Associates.

Woolfolk, A. (2000). Psicologia da Educação. Porto Alegre, RS: Artmed.

Zampieri, M., \& Schelini, P. W. (2013a). O uso de medidas intelectuais na análise do monitoramento metacognitivo de crianças. Psicologia: Teoria e Pesquisa, 29, 81-88. doi:10.1590/S0102-37722013000200007

Zampieri, M., \& Schelini, P. W. (2013b). Monitoramento metacognitivo de crianças de acordo com o nível de desempenho em medidas de capacidade intelectual. Psico, 44, 280-287.

Zechmeister, E. B., \& Shaughnessy, J. J. (1980). When you know that you know and when you think that you know but you don't. Bulletin of the Psychonomic Society, 15(1), 41-44. doi:10.3758/BF03329756

Sobre as autoras

Márcia Akemi Fujie é psicóloga, mestre e doutoranda em Psicologia pela Universidade Federal de São Carlos. Trabalha na linha de Comportamento Social e Processos Cognitivos e recebe financiamento de pesquisa da CAPES.

marcia.fujie@gmail.com

Patrícia Waltz Schelini é psicóloga, mestre e doutora pela Pontifícia Universidade Católica de Campinas, e pós-doutora pela Universidade do Minho. É Professora Associada 1 do Departamento de Psicologia da Universidade Federal de São Carlos, onde ministra aulas na graduação e pós-graduação e desenvolve estudos sobre inteligência/cognição, metacognição e pensamento imaginativo. Recebe financiamento de pesquisa da CAPES, CNPq e FAPESP. patriciaws01@gmail.com

A contribuição de cada autora pode ser atribuída como se segue: Márcia Akemi Fujie e Patrícia Waltz Schelini contribuíram para a conceitualização, investigação e visualização do artigo; Márcia Akemi Fujie e Patrícia Waltz Schelini foram responsáveis pela obtenção de financiamento; Márcia Akemi Fujie e Patrícia Waltz Schelini fizeram a redação inicial do artigo (rascunho) e Márcia Akemi Fujie e Patrícia Waltz Schelini são as responsáveis pela redação final (revisão e edição).

As autoras agradecem à Coordenação de Aperfeiçoamento de Pessoal de Nível Superior (CAPES) pelo financiamento da pesquisa. 
Recebido em: 30/11/2017

Revisado em: 24/07/2018

Aceito em: 19/08/2018 